Research Article

\title{
Consensus of the Distributed Multiagent System with the Framework of the Small-World Network
}

\author{
Jinping Mou (D), Qinbin He $\mathbb{D}$, Zhile Xia $\mathbb{D}^{\mathbb{D}}$, and Jie Wang $(\mathbb{D}$ \\ School of the Electronics and Information Engineering, Taizhou University, Taizhou, Zhejiang 318000, China \\ Correspondence should be addressed to Jinping Mou; mjptougaozhuanyong@163.com
}

Received 4 August 2020; Revised 10 January 2021; Accepted 2 February 2021; Published 15 February 2021

Academic Editor: Adrian Neagu

Copyright ( 92021 Jinping Mou et al. This is an open access article distributed under the Creative Commons Attribution License, which permits unrestricted use, distribution, and reproduction in any medium, provided the original work is properly cited.

\begin{abstract}
This paper investigates the consensus problem of the distributed multiagent system (MAS) with the small-world framework. A distributed consensus protocol is provided for the node-to-node communication. According to an error between every two neighbor agents, several consensus criteria among the agents are obtained firstly. Then, consensus criteria are obtained via the diameter of the graph of the MAS. Finally, based on the small-world framework, the consensus criteria are obtained; also, the relations among the consensus, the diameter of the path in the small-world framework, and the errors of agents are disclosed. Finally, one numerical example shows the reliability of the proposed methods.
\end{abstract}

\section{Introduction}

In recent years, the coordination problem of the MAS has been drawing considerable attention due to its wide range of application domains $[1,2]$, such as swarming $[3,4]$, clustering $[5,6]$, and flocking $[7,8]$. Consensus refers to the fact that the state of each agent converges to a common value.

To solve the consensus problem, the main task is to design the distributed protocol for each agent, and all agents achieve the consensus via the local communications. Hence, the well-designed protocol and optimized topology are usually considered when we investigate the problem.

For different circumstances, we need an effective consensus protocol to ensure the system keeps the coordinative behave, and there are many related results about it, such as the first-order MAS [9-12], the second-order MAS [13, 14], the high-order MAS $[15,16]$, and the fractional-order MAS [17-21]. Combined with weights and inequalities, sufficient conditions for consistency between the leader node and the following node are studied for the first-order MAS in [9]. H. J. LeBlanc and X. Koutsoukos studied the standards of the first-order MAS and the higher-order MAS consistency [10]. Based on the conditions of spanning trees and strong connectivity in the orientation diagram, the timing consistency of the first-order nonlinear MAS is studied in [12], and the main conditions for MAS consistency are obtained: in the leader-following case, the topology should have a generated tree; in the absence of a reader node, the MAS topology is strongly connected. Based on the eigenvalues of the matrix, the controllability of the second-order MAS is studied in [13], and some equivalent conditions for the second-order MAS controllability are obtained. In [14], the tracking problem of the second-order nonlinear MAS with time-variable and multiple leader nodes is studied, and the proposed control protocol can effectively deal with the problems such as the disturbance and the unknown input of the reader node. Using self-triggering control and dynamic output feedback control methods, the consensus of the highorder MAS is studied, and the obtained results can improve the communication efficiency of the MAS [15].

In many applications, the topology of the MAS should be optimized; hence, the fixed topology and switching topology are considered frequently. For example, in [20], based on the switching topology, quasi-consistency of the MAS with competition and collaboration is studied, and the results show that, as long as the collaboration time is long enough, the system can achieve the consensus. In addition, with the Lipschitz conditions, the adaptive consensus of the MAS under fixed topology is studied in [21]. Additionally, state estimation problems with Markovian switching are 
considered in $[22,23]$, and the fixed-time coordination problems are considered in [24-26].

Furthermore, by optimizing the topology of the MAS, the consensus problems of the MAS are further studied. Considering that the power system is affected by actuator saturation, the event trigger and self-trigger strategy are proposed, and the obtained results can save the resources of the network effectively [27].

Notice that most of the existed results about the consensus problems of the MAS are based on the fixed node set, and when the consensus is considered, the states of all agents are taken into account. In fact, in some applications, it is difficult to obtain all the information of the MAS, such as the $B A$ model of scale-free network (SFN) [28], and the newly added node is connected to the related original node with a certain probability. Therefore, by comparing the error between every two nodes on a route of the communication graph, the related problems have been considered. Based on the balance between the in-degree and the out-degree, the consensus problem has been studied in [29]. In the noise environment, the consensus problem of the MAS under the SFN structure is studied in [30], and the results show that noise has limited influence on system consistency; in addition, under the SFN topology, it shows that the system has strong robustness. According to an adaptive consensus protocol, the adaptive consensus problem of the MAS with the SFN structure is studied in [31], and several conditions of consensus are obtained.

In the last decade, the properties of small-world networks have been studied in [32-34]. A small-world network refers to an ensemble of networks in which the mean geodesic (i.e., shortest-path) distance between nodes increases sufficiently slowly as a function of the number of nodes in the network [35]. Based on the framework of the small-world network, many problems of different research fields have been solved, such as the efficiency issue of computing and maintaining the eccentricity distribution on a large dynamic network [36], the viral-style information diffusion [37], and the visual analytics [38]. Furthermore, the coordination problem has been considered in the smallworld network. In [39], the statistical properties of the consensus and synchronization of the small-world networks are studied, and the results show that the Cheeger constant, which is a major determinant to measure the convergence rate of the consensus and synchronization of the small-world networks, is investigated.

In fact, there is another way to study the consensus problem in the small-world framework. That is, by comparing the error between every two agents in the smallworld framework, the related consensus protocol is designed, and less error will be influenced, whereas all errors should be considered in the traditional results. Motivated by the idea, in this paper, we investigate the consensus problem in the small-world framework.

The remainder of this article is organized as the following. Section 2 introduces some notations and concepts. Section 3 proposes the consensus problem. In Section 4, several consensus criteria are obtained for the MAS. In Section 5, a numerical example shows the reliability of the proposed methods. In Section 6, we get the conclusions.

\section{Preliminaries}

Let $\mathscr{V}=\left\{v_{i} \mid i \in N\right\}$ be an agent set and $\mathscr{E}$ be the edge set, where $N$ is the natural number set.

Let $\mathscr{A}=\left(a_{i j}\right)_{N \times N}$ be a weighted symmetric adjacent matrix, where $a_{i j}$ is the weight between nodes $v_{i}$ and $v_{j}$, $a_{i j}=a_{j i}$, and $a_{i j}$ takes values of 1 or 0 . If $a_{i j}=1$, then it shows that there exists information flow between agents $v_{i}$ and $v_{j}$; or else, $a_{i j}=0$.

Let $\mathscr{E}=\left\{(i, j) \mid a_{i j}=1, i \neq j\right\}$ be the edge set, where $(i, j)$ refers to an edge between $v_{i}$ and $v_{j}$. $N_{i}$ is the neighbor set of $v_{i}$, where $N_{i}=\left\{j \mid\left(v_{i}, v_{j}\right) \in \mathscr{E}\right\}$. Then, the undirected weighted graph of a multiagent system (MAS) can be shown by $\mathscr{G}=(\mathscr{V}, \mathscr{E}, \mathscr{A})$.

$\forall v_{i} \in \mathscr{V}$, let $x_{i}(t) \in R^{n}$ be the state of agent $v_{i}$, where $t \in R$, and the consensus of the MAS can be interpreted as follows.

Definition 1 (see [31]). $\forall v_{i}, v_{j} \in \mathscr{V}$, if

$$
\lim _{t \longrightarrow \infty}\left\|x_{i}(t)-x_{j}(t)\right\|=0,
$$

then the system achieves the consensus, where $\left\|x_{i}(t)-x_{j}(t)\right\|$ is the norm of function $x_{i}(t)-x_{j}(t)$.

\section{Problem Statement}

Consider a MAS with the node set $\mathscr{V}$; the state of each node satisfies

$$
x_{i}^{\prime}(t)=u_{i}(t), \quad i \in N,
$$

where $u_{i}(t)$ is the consensus protocol of node $v_{i}$.

If $u_{i}(t)$ is implemented as

$$
u_{i}(t)=\sum_{j \in N_{i},\left\|x_{j}(t)\right\|>0} \frac{a_{i j}}{d_{i}}\left[\frac{c_{i}(t)\left\|x_{i}(t)\right\| x_{j}(t)}{\left\|x_{j}(t)\right\|}-x_{i}(t)\right]-\sum_{j \in N_{i},\left\|x_{j}(t)\right\|=0} \frac{a_{i j}}{d_{i}} x_{i}(t), \quad v_{i} \in \mathscr{V},
$$

where $d_{i}=\sum_{j \in N_{i}} a_{i j}$ and $c_{i}(t)$ is a function, then we have the following system: 


$$
x_{i}^{\prime}(t)=\sum_{j \in N_{i},\left\|x_{j}(t)\right\|>0} \frac{a_{i j}}{d_{i}}\left[\frac{c_{i}(t)\left\|x_{i}(t)\right\| x_{j}(t)}{\left\|x_{j}(t)\right\|}-x_{i}(t)\right]-\sum_{j \in N_{i},\left\|x_{j}(t)\right\|=0} \frac{a_{i j}}{d_{i}} x_{i}(t), \quad v_{i} \in \mathscr{V},
$$

or in a compact form,

$$
X^{\prime}(t)=[A(t)-I] X(t),
$$

where $X(t)=\left[x_{1}^{T}(t), x_{2}^{T}(t), \ldots\right]^{T}, I=\operatorname{diag}\{1,1, \ldots, 1, \ldots\}$, and

$$
\begin{aligned}
& A(t)=\left[\begin{array}{cccc}
b_{11}(t) & b_{12}(t) & b_{13}(t) & \cdots \\
b_{21}(t) & b_{22}(t) & b_{23}(t) & \ldots \\
\vdots & \ddots &
\end{array}\right], \\
& d_{i}^{1}=\sum_{j \in N_{i},\left\|x_{j}(t)\right\|>0} a_{i j}, \\
& d_{i}^{2}=\sum_{j \in N_{i},\left\|x_{j}(t)\right\|=0} a_{i j}, \\
& d_{i}=d_{i}^{1}+d_{i}^{2}, \\
& b_{i j}(t)= \begin{cases}\frac{1}{d_{i}^{1}} \frac{\left\|x_{i}(t)\right\|}{\left\|x_{j}(t)\right\|}, & \left\|x_{j}(t)\right\| \neq 0 \text { and } i \neq j, \\
0, & i=j .\end{cases}
\end{aligned}
$$

In the following sections, we are going to discuss the consensus of system (4), and we need the following assumption.

Assumption 1. We assume that $\forall v_{i}, v_{q} \in \mathscr{V}$, there exist two positive functions $c_{i}(t)$ and $\varepsilon_{i q}$ such that if $\left\|x_{i}(t)\right\| \neq\left\|x_{q}(t)\right\|$, then

$$
1>\varepsilon_{i q}>c_{i}(t) \frac{\left\|x_{i}(t)\right\|^{2}-2\left\|x_{i}(t)\right\|\left\|x_{q}(t)\right\| \cos \theta_{i q}+\left\|x_{q}(t)\right\|^{2}}{\left(\left\|x_{i}(t)\right\|-\left\|x_{i}(t)\right\|\right)^{2}},
$$

where

$$
\begin{aligned}
\cos \theta_{i q} & =\frac{x_{q}(t) x_{i}(t)}{\left\|x_{q}(t)\right\|\left\|x_{i}(t)\right\|}, \\
c_{i}(t) & = \begin{cases}\min _{v_{i} \in N_{j} \in \mathscr{V}} \frac{\left(\left\|x_{i}(t)\right\|-\left\|x_{i}(t)\right\|\right)^{2}}{\left(\left\|x_{i}(t)\right\|^{2}-2\left\|x_{i}(t)\right\|\left\|x_{j}(t)\right\| \cos \theta_{i j}+\left\|x_{j}(t)\right\|^{2}\right)}, & x_{i}(t) \neq x_{j}(t), \\
1, & x_{i}(t)=x_{j}(t) .\end{cases}
\end{aligned}
$$

Remark 1. If $\left\|x_{i}(t)\right\|=\left\|x_{q}(t)\right\|=0$, then the error between $v_{i}(t)$ and $v_{j}(t)$ is zero, and they achieve the consensus. If one of $\left\|x_{i}(t)\right\|$ and $\left\|x_{q}(t)\right\|$ is zero, then their angle of intersection is an arbitrary angle, and we define $\cos \theta_{i q}=1$.

It shows that the real networks have the following property; for any two nodes in a network, the distance between them is relatively small, while, at the same time, the level of transitivity or clustering is relatively high [40]. This property, which is shared by many real-world networks, is called the small-world phenomenon. In the following sections, based on the small-world framework of graph $\mathscr{G}$, the consensus problems of system (5) will be studied.

\section{The Main Results}

In this section, the consensus problem of the MAS is studied. Firstly, we propose the state error between every two neighbor agents. Then, in the connected topology, the state error between every two agents is provided via the route which associates with two agents. Finally, according to the diameter of a graph, consensus criteria are obtained; 
meanwhile, as a special case, consensus criteria are obtained for the small-world framework.

Theorem 1. If $q \in N_{i}$, then under protocol (5),

$$
\lim _{t \longrightarrow \infty}\left[x_{i}(t)-x_{q}(t)\right]=0,
$$

where $\cos \theta_{i q}=\left(\left(x_{q}(t) x_{i}(t)\right) /\left(\left\|x_{q}(t)\right\|\left\|x_{i}(t)\right\|\right)\right)$.

Proof. First, we estimate the error between $x_{i}(t)$ and $x_{i}(t)$ under the condition $\left\|x_{i}(t)\right\| \cdot\left\|x_{i}(t)\right\| \neq 0$. Since

$$
\begin{aligned}
& x_{i}^{\prime}(t)=\sum_{j \in N_{i},\left\|x_{j}(t)\right\|>0} \frac{a_{i j}}{d_{i}}\left[\frac{c_{i}(t)\left\|x_{i}(t)\right\| x_{j}(t)}{\left\|x_{j}(t)\right\|}-x_{i}(t)\right]-\sum_{j \in N_{i},\left\|x_{j}(t)\right\|=0} \frac{a_{i j}}{d_{i}} x_{i}(t), \\
& x_{q}^{\prime}(t)=\sum_{v \in N_{q},\left\|x_{v}(t)\right\|>0} \frac{a_{q v}}{d_{q}}\left[\frac{c_{q}(t)\left\|x_{q}(t)\right\| x_{v}(t)}{\left\|x_{v}(t)\right\|}-x_{q}(t)\right]-\sum_{v \in N_{q},\left\|x_{v}(t)\right\|=0} \frac{a_{q v}}{d_{i}} x_{q}(t),
\end{aligned}
$$

it holds that

$$
\begin{aligned}
x_{i}^{\prime}(t)-x_{q}^{\prime}(t) & =\sum_{j \in N_{i}} \frac{1}{d_{i}}\left[\frac{c_{i}(t)\left\|x_{i}(t)\right\| x_{j}(t)}{\left\|x_{j}(t)\right\|}-x_{i}(t)\right]-\sum_{v \in N_{q}} \frac{1}{d_{q}}\left[\frac{c_{q}(t)\left\|x_{q}(t)\right\| x_{v}(t)}{\left\|x_{v}(t)\right\|}-x_{q}(t)\right], \\
& =-\left[x_{i}(t)-x_{q}(t)\right]+\sum_{j \in N_{i}} \frac{1}{d_{i}} \frac{c_{i}(t)\left\|x_{i}(t)\right\| x_{j}(t)}{\left\|x_{j}(t)\right\|}-\sum_{v \in N_{q}} \frac{1}{d_{q}} \frac{c_{q}(t)\left\|x_{q}(t)\right\| x_{v}(t)}{\left\|x_{v}(t)\right\|} .
\end{aligned}
$$

For convenience, denote

$$
\begin{aligned}
& \delta_{i q}(t)=x_{i}(t)-x_{q}(t), \\
& f_{i q}(t)=\sum_{j \in N_{i}} \frac{1}{d_{i}} \frac{c_{i}(t)\left\|x_{i}(t)\right\| x_{j}(t)}{\left\|x_{j}(t)\right\|}-\sum_{v \in N_{q}} \frac{1}{d_{q}} \frac{c_{q}(t)\left\|x_{q}(t)\right\| x_{v}(t)}{\left\|x_{v}(t)\right\|} .
\end{aligned}
$$

We have

$$
\begin{aligned}
\left\|f_{i q}(t)\right\| & \leq\left\|\sum_{j \in N_{i}} \frac{1}{d_{i}} \frac{c_{i}(t)\left\|x_{i}(t)\right\| x_{j}(t)}{\left\|x_{j}(t)\right\|}-\sum_{v \in N_{q}} \frac{1}{d_{q}} \frac{c_{q}(t)\left\|x_{q}(t)\right\| x_{v}(t) \|,}{\left\|x_{v}(t)\right\|}\right\| \\
& =\| \sum_{j \in N_{i}, v \in N_{q}}\left[\frac{1}{d_{q} d_{i}} \frac{c_{i}(t)\left\|x_{i}(t)\right\| x_{j}(t)}{\left\|x_{j}(t)\right\|}-\frac{1}{d_{q} d_{i}} \frac{c_{q}(t)\left\|x_{q}(t)\right\| x_{v}(t)}{\left\|x_{v}(t)\right\|}\right], \\
& \leq \sum_{j \in N_{i}, v \in N_{q}} \frac{1}{d_{q} d_{i}}\left\|\frac{c_{i}(t)\left\|x_{i}(t)\right\| x_{j}(t)}{\left\|x_{j}(t)\right\|}-\frac{c_{q}(t)\left\|x_{q}(t)\right\| x_{v}(t) \|}{\left\|x_{v}(t)\right\|}\right\| .
\end{aligned}
$$

Let $c(t)=\max \left\{c_{i}(t), c_{q}(t)\right\}$; it follows that 


$$
\begin{aligned}
\left\|f_{i q}(t)\right\| & \leq \sum_{j \in N_{i}, v \in N_{q}} \frac{c(t)}{d_{q} d_{i}}\left(\sqrt{\frac{\left\|x_{i}(t)\right\| x_{j}(t)}{\left\|x_{j}(t)\right\|}-\frac{\left\|x_{q}(t)\right\| x_{v}(t)}{\left\|x_{v}(t)\right\|}}\right)^{2} \\
& \leq \sum_{j \in N_{i}, v \in N_{q}} \frac{c_{i}(t)}{d_{q} d_{i}} \sqrt{\left\|x_{i}(t)\right\|^{2}-2 \frac{\left\|x_{i}(t)\right\| x_{j}(t)}{\left\|x_{j}(t)\right\|} \frac{\left\|x_{q}(t)\right\| x_{v}(t)}{\left\|x_{v}(t)\right\|}+\left\|x_{q}(t)\right\|^{2}} \\
& =\sum_{j \in N_{i}, v \in N_{q}} \frac{1}{d_{q} d_{i}} \sqrt{c_{i}(t)^{2}\left[\left\|x_{i}(t)\right\|^{2}-2\left\|x_{i}(t)\right\|\left\|x_{q}(t)\right\| \cos \theta_{j v}+\left\|x_{q}(t)\right\|^{2}\right]} .
\end{aligned}
$$

From Assumption 1, it holds that

$$
\frac{\varepsilon_{i q}-c(t)^{2}}{\varepsilon_{i q}-c(t)^{2} \cos \theta_{i q}}>\frac{2\left\|x_{i}(t)\right\|\left\|x_{q}(t)\right\|}{\left\|x_{i}(t)\right\|^{2}+\left\|x_{q}(t)\right\|^{2}},
$$

and it follows that

$$
\varepsilon_{i q}\left(\left\|x_{i}(t)\right\|^{2}+2\left\|x_{i}(t)\right\|\left\|x_{q}(t)\right\|+\left\|x_{q}(t)\right\|^{2}\right)>c_{i}(t)^{2}\left(\left\|x_{i}(t)\right\|^{2}+2\left\|x_{i}(t)\right\|\left\|x_{q}(t)\right\| \cos \theta_{i q}+\left\|x_{q}(t)\right\|^{2}\right) .
$$

Hence, we have

$$
\begin{aligned}
\left\|f_{i q}(t)\right\| & <\sum_{j \in N_{i}, v \in N_{q}} \frac{c}{d_{q} d_{i}} \sqrt{c_{i}(t)^{2}\left[\left\|x_{i}(t)\right\|^{2}-2\left\|x_{i}(t)\right\|\left\|x_{q}(t)\right\|+\left\|x_{q}(t)\right\|^{2}\right]}, \\
& \leq \varepsilon_{i q} \sum_{j \in N_{i}, v \in N_{q}} \frac{1}{d_{q} d_{i}}\left[\left\|x_{i}(t)\right\|-\left\|x_{q}(t)\right\|\right] \\
& \leq \varepsilon_{i q} \sum_{j \in N_{i}, v \in N_{q}} \frac{1}{d_{q} d_{i}}\left\|x_{i}(t)-x_{q}(t)\right\|, \\
& \leq \varepsilon_{i q}\left\|x_{i}(t)-x_{q}(t)\right\| .
\end{aligned}
$$

Let the Lyapunov candidate be
$V_{i q}(t)=(1 / 2) \delta_{i q}(t)^{T} \delta_{i q}(t) ;$ then, we get

$$
\begin{aligned}
V_{i q}^{\prime}(t) & =\frac{1}{2}\left[\delta_{i q}^{\prime}(t)^{T} \delta_{i q}(t)+\delta_{i q}(t)^{T} \delta_{i q}^{\prime}(t)\right] \\
& \leq \frac{1}{2}\left[-\delta_{i q}(t)+f_{i q}(t)\right]^{T} \delta_{i q}(t)+\delta_{i q}(t)^{T}\left[-\delta_{i q}(t)+f_{i q}(t)\right], \\
& \leq-\delta_{i q}(t)^{T} \delta_{i q}(t)+\frac{1}{2}\left[f_{i q}(t)\right]^{T} \delta_{i q}(t)+\delta_{i q}(t)^{T} f_{i q}(t), \\
& \leq-\delta_{i q}(t)^{T} \delta_{i q}(t)+\varepsilon_{i q}\left\|x_{i}(t)-x_{q}(t)\right\|\left\|\delta_{i q}(t)\right\|, \\
& =-\left\|\delta_{i q}(t)\right\|^{2}+\varepsilon_{i q}\left\|\delta_{i q}(t)\right\|^{2}, \\
& =2\left(-1+\varepsilon_{i q}\right) V_{i q}(t), \\
& <0,
\end{aligned}
$$

and $\lim _{t \longrightarrow \infty} \delta_{i q}(t)=0$, namely, $\lim _{t \longrightarrow \infty}\left[x_{i}(t)-x_{q}(t)\right]=0$. 
$\left\|x_{q}(t)\right\|=0$, namely, $x_{q}(t)$ is the zero vector, and we can get the same result. This completes the proof.

Theorem 2. If $\mathscr{G}$ is connected, then under protocol (5), system (4) achieves the consensus.
Proof. If $q \in N_{i}$, then $\lim _{t \rightarrow \infty}\left[x_{i}(t)-x_{q}(t)\right]=0$ has been proved in Theorem 1.

Since $\mathscr{G}$ is connected, if $q \notin N_{i}$, then there exists a path $v_{i} v_{l_{1}} v_{l_{2}}, \ldots, v_{l_{n}} v_{q}$ between $v_{i}$ and $v_{q}$ such that

$$
\lim _{t \longrightarrow \infty}\left\|x_{i}(t)-x_{q}(t)\right\| \leq \lim _{t \longrightarrow \infty}\left\|x_{i}(t)-x_{l_{1}}(t)\right\|+\lim _{t \longrightarrow \infty}\left\|x_{l_{1}}(t)-x_{2}(t)\right\|+\cdots+\lim _{t \longrightarrow \infty}\left\|x_{l_{n}}(t)-x_{q}(t)\right\|=0,
$$

and on the contrary, $\lim _{t}\left\|x_{i}(t)-x_{q}(t)\right\| \geq 0$; then, we have $\lim _{t \rightarrow \infty}\left\|x_{i}(t)-x_{q}(t)\right\| \stackrel{t \overrightarrow{=}}{=}$, and this completes the proof.

Remark 2. The consensus criterion of Theorem 2 is a rough result; it has not disclosed the relations among the scale of the network, the bound of tolerate errors, and the diameter of the graph $G$. In the following results, the relations will be discussed.

Assumption 2. For each diameter $P=v_{i_{1}} v_{i_{1}} \ldots v_{i_{s}} \ldots v_{i_{n+1}}$ of graph $\mathscr{G}$, we assume that there exist two functions $V_{i M}(t)$ and $V_{i m}(t)$ such that

$$
V_{i M} \geq V_{i q}(t) \geq V_{\text {im }}
$$

where

$$
V_{i q}(t)=(1 / 2) \delta_{i q}^{T} \delta_{i q}(t)
$$

$v_{q} \in N_{i}$ $i, q \in\left\{v_{i_{1}}, v_{i_{1}}, \ldots, v_{i_{s}}, \ldots, v_{i_{n}}\right\}$, and $V_{i M}$ and $V_{i m}$ are nonnegative constants.

Theorem 3. If $P=v_{i 1} v_{i 2} \ldots v_{i(L+1)}$ is the diameter of graph $\mathscr{G}$ and $G$ is connected, then under the control of protocol (5), system (4) achieves the consensus if

$$
\sum_{l=1}^{L} \varepsilon_{i_{i} i_{l+1}}<1,
$$

where $L$ is the length of path $i_{1} i_{2} \ldots i_{L} i_{q}$ in the small-world network of the MAS.

Proof. Let $V_{i q}(t)=(1 / 2) \delta_{i} i_{2}{ }^{T}(t) \delta_{i_{1} i_{2}}(t)+(1 / 2) \delta_{i_{2} i_{3}}^{T}(t) \delta_{i_{2} i_{3}}$ $(t)+\cdots+(1 / 2) \delta_{i_{L} i_{L+1}}^{T}(t) \delta_{i_{L} i_{L+1}}(t)$; then, it holds that

$$
\begin{aligned}
V_{i_{1} i_{L+1}}{ }^{\prime}(t) & =\frac{1}{2}\left[\delta_{i_{1} i_{2}}^{\prime T}(t) \delta_{i_{1} i_{2}}(t)+\delta_{i_{1} i_{2}}^{T}(t) \delta_{i_{1} i_{2}}^{\prime}(t)+\cdots+\delta_{i_{L} i_{L+1}}^{\prime T}(t) \delta_{i_{L} i_{L+1}}(t)+\delta_{i_{L} i_{L+1}}^{T}(t) \delta_{i_{L} i_{L+1}}{ }^{\prime}(t)\right], \\
& \leq \frac{1}{2}\left\{\left[-\delta_{i_{1} i_{2}}(t)+f_{i_{1} i_{2}}(t)\right]^{T} \delta_{i_{1} i_{2}}(t)+\cdots+\delta_{i_{L} i_{L+1}}(t)^{T}\left[-\delta_{i_{L} i_{L+1}}(t)+f_{i_{L} i_{L+1}}(t)\right],\right. \\
& \left.+\delta_{i_{1} i_{2}}^{T}(t)\left[-\delta_{i_{1} i_{2}}(t)+f_{i_{1} i_{2}}(t)\right]+\cdots+\left[-\delta_{i_{n} i_{L+1}}(t)+f_{i_{n} i_{L+1}}(t)\right]^{T} \delta_{i_{n} i_{L+1}}(t)\right\}, \\
& \leq-\delta_{i_{1} i_{L+1}}(t)^{T} \delta_{i_{1} i_{L+1}}(t)+\left[f_{i_{1} i_{2}}(t)\right]^{T} \delta_{i_{1} i_{2}}(t)+\cdots+\delta_{i_{n} i_{L+1}}(t)^{T} f_{i_{L} i_{L+1}}(t), \\
& \leq-\delta_{i q}(t)^{T} \delta_{i q}(t)+\varepsilon_{i_{1} i_{2}}\left\|x_{i_{1}}(t)-x_{i_{2}}(t)\right\|\left\|\delta_{i_{1} i_{2}}(t)\right\|+\cdots+\varepsilon_{i q}\left\|x_{i_{n}}(t)-x_{i_{L+1}}(t)\right\|\left\|\delta_{i_{n} i_{L+1}}(t)\right\|, \\
& \leq-\delta_{i q}(t)^{T} \delta_{i q}(t)+\varepsilon_{i q}\left[\varepsilon_{i_{1} i_{2}} \delta_{i_{1} i_{2}}(t)^{T} \delta_{i_{1} i_{2}}(t)+\cdots+\varepsilon_{i_{L} i_{L+1}} \delta_{i_{L} i_{L+1}}(t)^{T} \delta_{i_{L} i_{L+1}}(t)\right], \\
& =2\left[-V_{i q}+V_{i q}\right]=0,
\end{aligned}
$$

and it follows that $\forall v_{i}, v_{q} \in \mathscr{V}, \lim _{t \longrightarrow \infty}\left\|x_{i}(t)-x_{q}(t)\right\|=0$. This completes the proof.

Corollary 1. If $P=v_{i 1} v_{i 2} \ldots v_{i(L+1)}$ is the diameter of a smallworld graph $\mathscr{G}, G$ is connected, and $V_{i m}(t)=m>0$ and $V_{i M}(t)=M>0$, then under the control of protocol (5), system (4) achieves the consensus if $\forall v_{i}, v_{q} \in \mathscr{V}$,

$$
0<\varepsilon_{i q}<\frac{m}{L M} \text {. }
$$

Proof. According to the proof of Theorem 3, it holds that $V_{i q}^{\prime}(t) \leq 2\left[-V_{i m}+\varepsilon_{i q} L V_{i M}\right]<0$.

Hence, system (4) achieves consensus, and this completes the proof.

In this section, the consensus problem of the MAS has been studied. In the next section, a numerical example shows the reliability of the obtained methods. 


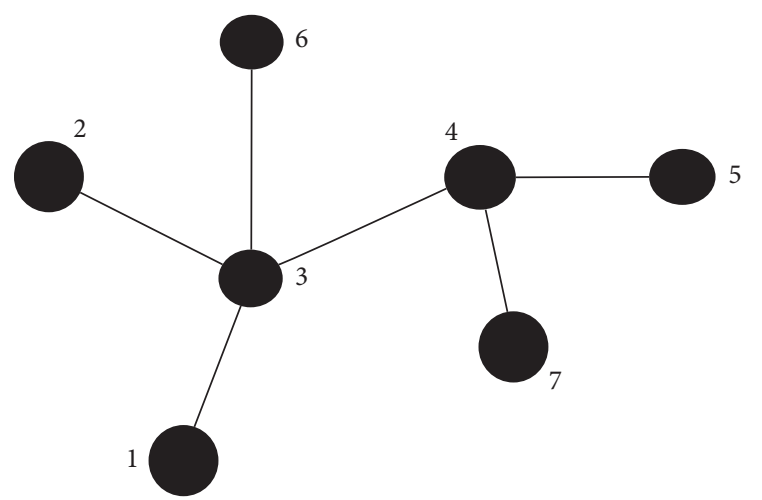

Figure 1: The topology of MAS (4).

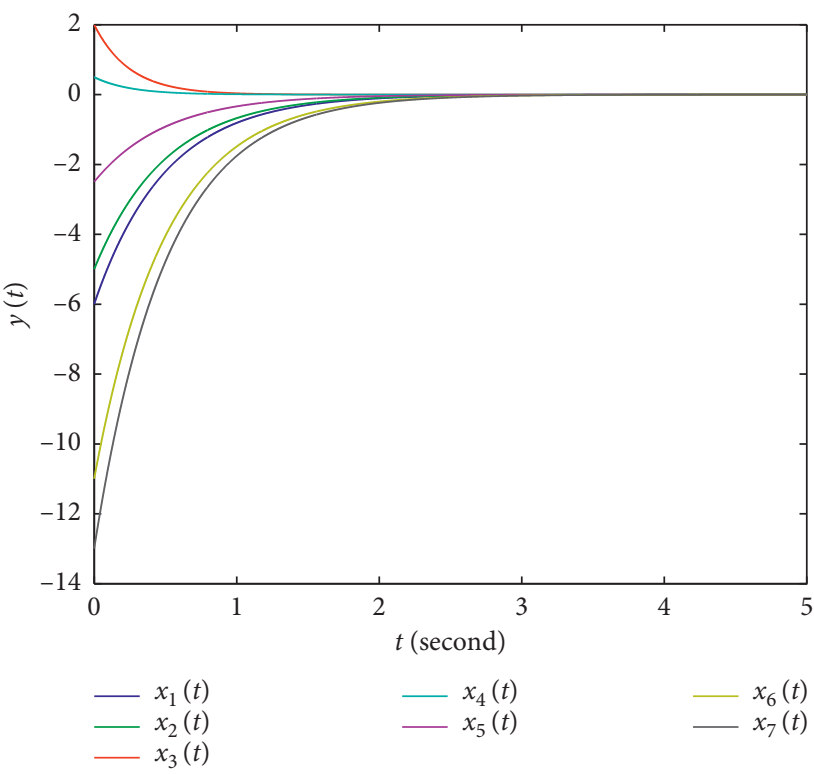

Figure 2: All agents of MAS (4) achieve the consensus.

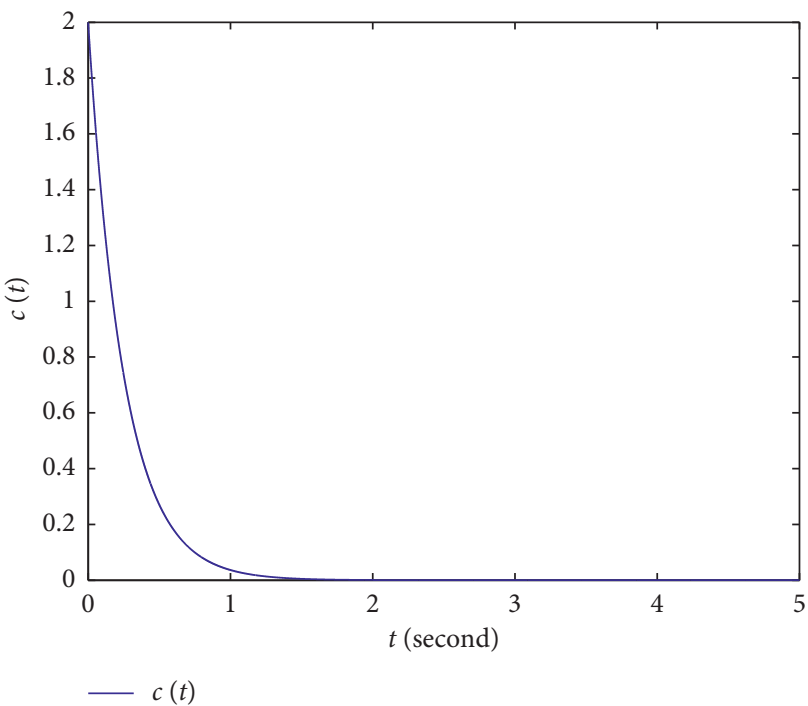

FIgURe 3: Function of $c(t)$.

\section{A Numerical Example}

Example 1. Consider a connected MAS (4) with seven agents, where $\mathscr{V}=\left\{v_{1}, v_{2}, v_{3}, v_{4}, v_{5}, v_{6}, v_{7}\right\}$, and the topology is shown in Figure 1. Suppose $x_{i}(t) \in R$, let

$$
\varepsilon_{i, q}=\frac{1}{4} c_{i}(t) \frac{\left\|x_{i}(t)\right\|^{2}-2\left\|x_{i}(t)\right\|\left\|x_{q}(t)\right\| \cos \theta_{i q}+\left\|x_{q}(t)\right\|^{2}}{\left(\left\|x_{i}(t)\right\|-\left\|x_{q}(t)\right\|\right)^{2}},
$$

where $i, q=1,2, \ldots, 7$, and according to Corollary 1 , under the control of protocol (3), MAS (5) achieves the consensus. The simulation result is shown in Figures 2 and 3, where $c(t)=\max \left\{c_{i}(t) \mid i=1,2, \ldots, 7\right\}$.

\section{Conclusion}

In this paper, the consensus problem of the MAS has been investigated via the node-to-node communications. A kind of distributed protocol has been designed firstly. Then, we have obtained a system with scale-free topology. By the theoretical analysis, the results have shown that based on the protocols, all agents can achieve the consensus; meanwhile, a criterion on the small-world framework has been obtained. Finally, the numerical example has shown the reliability of the proposed methods.

\section{Data Availability}

The data used to support the findings of this study are available from the corresponding author upon request.

\section{Conflicts of Interest}

The authors declare that they have no conflicts of interest.

\section{Acknowledgments}

This work is supported by Fund of Zhejiang Provincial Education Department (Y201941722), the Cultivation Funds of Taizhou University (2019PY017 and 2017PY17), and Research Initiation Fund of Taizhou University (0104010004).

\section{References}

[1] F. Chen and W. Ren, "On the control of multi-agent systems: a survey (Review)," Foundations and Trends in Systems and Control, vol. 6, no. 4, pp. 1-164, 2019.

[2] V. S. Kozyakin, N. A. Kuznetsov, and P. Y. Chebotarev, "Consensus in asynchronous multiagent systems. III. Constructive stability and stabilizability," Automation and Remote Control, vol. 80, no. 6, pp. 989-1015, 2019.

[3] F. Wang, H. Yang, and Y. Yang, "Swarming movement of dynamical multi-agent systems with sampling control and time delays," Soft Computing, vol. 23, no. 2, pp. 707-714, 2019.

[4] Y. Wan, J. Tang, and S. Lao, "Distributed conflict-detection and resolution algorithm for UAV swarms based on consensus algorithm and strategy coordination," IEEE Access, vol. 7, pp. 100552-100566, 2019. 
[5] X. Qiu, Y. Zhang, and K. Li, "Successive lag cluster consensus on multi-agent systems via delay-dependent impulsive control," Chinese Physics B, vol. 28, no. 5, Article ID 050501, 2019.

[6] X. Guo, J. Liang, and H. M. Fardoun, "New methods to realize the cluster consensus for multi-agent networks," Asian Journal of Control, vol. 22, no. 6, p. 2549, 2019.

[7] W. Zhao, H. Chu, M. Zhang, T. Sun, and L. Guo, "Flocking control of fixed-wing UAVs with cooperative obstacle avoidance capability," IEEE Access, vol. 7, pp. 17798-17808, 2019.

[8] S. Su and Z. Lin, "Connectivity enhancing coordinated tracking control of multi-agent systems with a state-dependent jointly-connected dynamic interaction topology," Automatica, vol. 101, pp. 431-438, 2019.

[9] Y. Gao and Y. Zhang, "Consensus of first-order multi-agent systems based on cooperation and competition," in Proceedings of the 2018 Chinese Control And Decision Conference (CCDC), pp. 6089-6093, Shenyang, China, June 2018.

[10] H. J. LeBlanc and X. Koutsoukos, "Resilient first-order consensus and weakly stable, higher order synchronization of continuous-time networked multiagent systems," IEEE Transactions on Control of Network Systems, vol. 5, no. 3, pp. 1219-1231, 2018.

[11] D. Tong, C. Xu, Q. Chen, and W. Zhou, "Sliding mode control of a class of nonlinear systems," Journal of the Franklin Institute, vol. 357, no. 3, pp. 1560-1581, 2020.

[12] H. Wang, W. Yu, G. Wen, and G. Chen, "Fixed-time consensus of nonlinear multi-agent systems with general directed topologies," IEEE Transactions on Circuits and Systems II: Express Briefs, vol. 66, no. 9, pp. 1587-1591, 2019.

[13] M. Long, H. Su, and B. Liu, "Second-order controllability of two-time-scale multi-agent systems," Applied Mathematics and Computation, vol. 343, no. 1, pp. 299313, 2019.

[14] J. Yu, X. Dong, Q. Li, and Z. Ren, "Practical time-varying formation tracking for second-order nonlinear multiagent systems with multiple leaders using adaptive neural networks," IEEE Transactions on Neural Networks and Learning Systems, vol. 29, no. 12, pp. 6015-6025, 2018.

[15] X. You, C. Hua, and X. Guan, "Self-Triggered leader-following consensus for high-order nonlinear multiagent systems via dynamic output feedback control," IEEE Transactions on Cybernetics, vol. 49, no. 6, pp. 2002-2010, 2019.

[16] X. Lin, X. Li, C.-C. Chen, and S. Li, "Smooth output feedback stabilization for a class of high-order switched nonlinear systems," Nonlinear Analysis: Hybrid Systems, vol. 29, no. 1, pp. 34-53, 2018.

[17] H. Yang, Y. Yang, F. Han et al., "Containment control of heterogeneous fractional-order multi-agent systems," Journal of the Franklin Institute, vol. 356, no. 12, pp. 752-765, 2019.

[18] M. Tabatabaei, "Leader-following consensus of discrete-time fractional-order multi-agent systems," Chinese Physics B, vol. 27, no. 1, Article ID 010701, 2018.

[19] L. Long, "Integral ISS for switched nonlinear time-varying systems using indefinite multiple Lyapunov functions," IEEE Transactions on Automatic Control, vol. 64, no. 1, pp. 404-411, 2019.

[20] W. Zhang, D. W. C. Ho, Y. Tang, and Y. Liu, "Quasi-consensus of heterogeneous-switched nonlinear multiagent systems," IEEE Transactions on Cybernetics, vol. 50, no. 7 , pp. 3136-3146, 2020.
[21] T. Zheng, J. Xi, M. Yuan, and G. Liu, "Adaptive consensualization for Lipschitz nonlinear-type multi-agent networks with fixed topologies," International Journal of Control, Automation and Systems, vol. 16, no. 4, pp. 1493-1501, 2018.

[22] D. Tong, C. Xu, Q. Chen, W. Zhou, and Y. Xu, "Sliding mode control for nonlinear stochastic systems with Markovian jumping parameters and mode-dependent time-varying delays," Nonlinear Dynamics, vol. 100, no. 2, pp. 1343-1358, 2020.

[23] D. Tong, Q. Chen, W. Zhou, and Y. Xu, "Adaptive state estimation of Markov switched neural networks driven by Lévy noise," Transactions of the Institute of Measurement and Control, vol. 42, no. 2, pp. 330-336, 2020.

[24] Y. Xu, X. Wu, N. Li, L. Liu, C. Xie, and C. Li, "Fixed-time synchronization of complex networks with a simpler nonchattering controller," IEEE Transactions on Circuits and Systems II: Express Briefs, vol. 67, no. 4, pp. 700-704, 2020.

[25] Y. Xu, X. Wu, and B. Mao, J. Lu, Fixed-time synchronization in the pth moment for time-varying delay stochastic multilayer networks," IEEE Transactions on Systems, Man, and Cybernetics: Systems, 2020, In press.

[26] Y. Xu, X. Wu, B. Mao, and C. Xie, "A unified finite-/fixed-time synchronization approach to multi-layer networks," IEEE Transactions on Circuits and Systems II: Express Briefs, vol. 68, no. 1, pp. 311-315, 2021.

[27] Z. Zuo, Q. Li, H. Li, and Y. Wang, "Event-triggered and selftriggered control for linear systems with actuator saturation," Transactions of the Institute of Measurement and Control, vol. 40, no. 6, pp. 1281-1288, 2018.

[28] L. Zhu, L. Wang, X. Zheng, and Y. Xu, "The Barabási and Albert scale-free network model," Journal of Intelligent \& Fuzzy Systems, vol. 35, no. 1, pp. 123-132, 2018.

[29] H. Wang and Y. Guo, "Consensus on scale-free network," in Proceedings of the 2008 Amercia Control Conference Westin Seattle Hotel, pp. 748-752, Seattle, WA, USA, June 2008.

[30] Y. Yi, Z. Zhang, and S. Patterson, "Scale-free loopy structure is resistant to noise in consensus dynamics in complex networks," IEEE Transactions on Cybernetics, vol. 50, no. 1, pp. 190-200, 2020.

[31] J. Mou, H. Ge, and J. Wang, "Adaptive consensus of scale-free multi-agent systems with event-triggered communications," International Journal of Modelling, Identification and Control, vol. 27, no. 2, pp. 154-162, 2017.

[32] L. Gu, H. L. Huang, and X. D. Zhang, "The clustering coefficient and the diameter of small-world networks," Acta Mathematica Sinica, English Series, vol. 29, no. 1, pp. 199-208, 2013.

[33] F. Ma and B. Yao, "The relations between network-operation and topological-property in a scale-free and smallworld network with community structure," Physica A: Statistical Mechanics and Its Applications, vol. 484, pp. 182-193, 2017.

[34] A. Ganesh and F. Xue, "On the connectivity and diameter of small-world networks," Advances in Applied Probability, vol. 39, no. 4, pp. 853-863, 2007.

[35] M. A. Porter, "Small-world network," Scholarpedia, vol. 7, no. 2, p. 1739, 2012.

[36] W. Li, M. Qiao, L. Qin, Y. Zhang, L. Chang, and X. Lin, "Eccentricities on small-world networks," The VLDB Journal, vol. 28, no. 5, pp. 765-792, 2019. 
[37] F. Li and Y. Wei, "Simulation analysis of viral-style information diffusion in small world networks," Journal of System Simulation, vol. 31, no. 9, pp. 1790-1801, 2019.

[38] C.-C. Lin, W. Huang, W.-Y. Liu, and S.-F. Wu, "A novel centrality-based method for visual analytics of small-world networks," Journal of Visualization, vol. 22, no. 5, pp. 973-990, 2019.

[39] L. Gu, X.-D. Zhang, and Q. Zhou, "Consensus and synchronization problems on small-world networks," Journal of Mathematical Physics, vol. 51, no. 8, Article ID 082701, 2010.

[40] D. Q. Nykamp, “Small-world networks," http://mathinsight. org/small-world network. 